\title{
Identificación morfológica y molecular de filarias caninas en una comuna semi-rural de la Región Metropolitana, Chile
}

\author{
Javier López, Fernando Valiente-Echeverría, Marcela Carrasco, Rubén Mercado y Katia Abarca
}

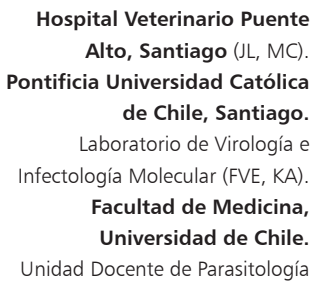

Hospital Veterinario Puente Alto, Santiago (JL, MC) Pontificia Universidad Católica de Chile, Santiago. Laboratorio de Virología e Infectología Molecular (FVE, KA) Facultad de Medicina, Universidad de Chile. Unidad Docente de Parasitología (RM).

Financiamiento: No hubo. Los autores no tienen conflictos de interés que declarar.

Recibido: 6 de junio de 2011 Aceptado: 23 de marzo de 2012

Correspondencia a: Katia Abarca Villaseca E-mail: katia@med.puc.cl.

\section{Morphological and molecular identification of canine filariae in a semi-rural district of the Metropolitan Region in Chile}

Introduction: To date, there has been no definitive confirmation of the presence of zoonotic dirofilariasis in dogs in Chile. Objectives: To study the presence of dirofilarias in blood samples from dogs collected in a semi-rural district near Santiago and to compare their frequency in dogs with and without dermatological manifestations. Methods: We examined 100 blood samples for dog filariae infections using microscopic methods (modified Knott technique). 50 dogs presented dermatological symptoms or signs compatible with filarial infections and 50 were asymptomatic. ITS-2 and 12s rDNA gene amplification by PCR and sequencing were performed in samples microscopically positive for microfilariae. Results. We observed microfilariae in 22 dogs $(22 \%)$. Of these, 16/50 $(32 \%)$ were symptomatic and $6 / 50(12 \%)$ were asymptomatic $(p=0.02)$. Morphologically, the majority of microfilariae were similar to Dirofilaria repens, although many had a bigger size than previously described. Nucleotide sequencing of the amplified genes showed no more than $95 \%$ homology with the $D$. repens sequences available for comparison. D. reconditum and $D$. dracunculoides infections were also identified. Conclusions: These features might indicate the presence of new species of Dirofilaria or a D. repens close related variant in Chile.

Key words: Canine filariasis, microfilariae, Dirofilaria repens, zoonotic dirofilariasis

Palabras clave: Filariasis canina, microfilaria, Dirofilaria repens, dirofilariasis zoonótica.

\section{Introducción}

L a dirofilariasis es una zoonosis emergente transmitida por mosquitos zoo-antropofílicos de la familia Culicidae. Los perros constituyen el principal hospedero y reservorio y pueden ser infectados por varias especies, siendo Dirofilaria immitis y Dirofilaria repens las involucradas en las infecciones zonóticas ${ }^{1-3}$. Ambas especies producen microfilaremia de larga duración en los perros. En el caso de D. immitis (conocida como el gusano del corazón de los perros), la afección es de localización pulmonar y cardíaca, en tanto que para D. repens es de ubicación subcutánea y conjuntival ${ }^{1,4}$. Además, en asociación a esta última especie, se han descrito una variedad de manifestaciones dermatológicas, tales como prurito, eritema, pápulas y alopecia, entre otras ${ }^{5,6}$. Las especies del género Dipetalonema, si bien se encuentran muy frecuentemente en los perros, tienen baja patogenicidad y no se han relacionado con infecciones en humanos.

Dirofilaria repens se encuentra presente en los continentes de Europa, Asia y África; siendo considerada una especie propia del Viejo Mundo ${ }^{1-4}$. Por el contrario, la distribución de $D$. immitis es más universal, abarcando todos los continentes, incluyendo varios países americanos ${ }^{1,4,7}$.

En Chile, un estudio realizado en perros de distintas ciudades del país reportó microfilaremias del género $\mathrm{Di}$ petalonema, especies D. reconditum y D. dracunculoides ${ }^{8}$, mientras que otro realizado posteriormente en la Región de Valparaíso encontró sólo D. reconditum ${ }^{9}$. Ninguno de estos estudios publicados, así como varias tesis no publicadas, ha reportado la presencia de $D$. immitis en Chile; asimismo, en la comunidad veterinaria no ha existido la sospecha clínica de esta afección en perros.

Uno de los principales artrópodos vectores de esta parasitosis, los mosquitos del género Culex, se encuentran ampliamente distribuidos en Chile continenta ${ }^{10}$. Otros vectores, particularmente de las especies de Dipetalome$n a$, son pulgas y la garrapata café del perro ${ }^{1}$.

En nuestro conocimiento, el único caso reportado de filariasis humana adquirido en Chile corresponde al de una niña de la comuna de Quilicura con un espécimen identificado como gusano filárido adulto extraído quirúrgicamente desde un nódulo subcutáneo del brazo ${ }^{11}$. Si bien no se diagnosticó la especie causal, su localización subcutánea sugiere que podría tratarse de $D$. repens.

El objetivo de este estudio fue investigar la presencia de dirofilarias en perros de una comuna semi-rural de la RM de Chile, utilizando por primera vez en el país métodos moleculares para su diagnóstico. Además, evaluar la relación de la presencia de microfilarias con síntomas dermatológicos. 


\section{Materiales y Métodos}

Población y muestras. El cálculo del tamaño de muestra considerando la prevalencia descrita por Alcaíno ${ }^{8}$ y un nivel de confianza de $95 \%$ arrojó 80 perros para un error absoluto de $10 \%$ y 100 perros para un error absoluto de $9 \%$.

Se tomó una muestra de sangre venosa a 100 perros de viviendas de sectores rurales de la comuna de Lampa $\left(33^{\circ} 17^{\prime} 0^{\prime \prime} \mathrm{S}, 70^{\circ} 54^{\prime} 0^{\prime \prime} \mathrm{W}\right)$; 50 de ellos eran asintomáticos y 50 tenían manifestaciones dermatológicas, tales como dermatitis, alopecia, seborrea o prurito.

Observación de las microfilarias. Las muestras de sangre se procesaron por la técnica de Knott modificada y fueron observadas al microscopio para determinar la presencia de microfilarias; la diferenciación de especies se basó en las características morfológicas de las microfilarias $^{12}$. Se registró el largo y el diámetro de 4-5 especímenes por muestra.

Extracción de ADN. La extracción de ADN se realizó según lo descrito previamente ${ }^{13} .300 \mu \mathrm{L}$ de cada muestra se mezclaron con $700 \mu \mathrm{L}$ de tampón I (10 mM Tris-HCl, pH 8,0, 1 mM EDTA, pH 8,0) y se centrifugó la mezcla. El pellet se lavó con tampón de lavado (10 mM Tris, $1 \mathrm{mM}$ EDTA, pH 8,0) y se resuspendió en $500 \mu \mathrm{L}$ de tampón de lisis de eritrocitos ( $1 \mathrm{M}$ sucrosa, $10 \mathrm{mM}$ Tris- $\mathrm{HCl}, \mathrm{pH} 7,5$, $5 \mathrm{mM} \mathrm{MgCl}_{2}, 1 \%$ Triton X-100). Luego de centrifugar, se eliminó el sobrenadante, se agregaron $200 \mu \mathrm{L}$ de tampón de lisis (20 mM Tris- $\mathrm{HCl}, \mathrm{pH} 8,0,50 \mathrm{mM} \mathrm{KCl}, 2,5 \mathrm{mM}$ $\mathrm{MgCl}_{2}, 0,5 \%$ Tween $20,150 \mu \mathrm{g} / \mathrm{mL}$ de proteinasa $\mathrm{K}$ ) y se incubó a $65^{\circ} \mathrm{C}$ por $3 \mathrm{~h}$. El ADN se purificó mediante extracción con fenol/cloroformo. El ADN extraído se resuspendió en $10 \mu \mathrm{L}$ de tampón $\mathrm{TE}^{13}$.

Amplificación y secuenciamiento. La identificación genérica de filarias se realizó mediante amplificación de la región espaciadora interna correspondiente al 5,8S y el 28S del ADN ribosomal (ITS-2); como control de extracción se amplificó el gen de $\beta$-actina canina. Los procedimientos de RPC se realizaron según lo descrito $^{13,14}$ usando partidores DIDR-F1/DIDR-R1 y actinaF2/ actinaR2 (Tabla 1). Para cada reacción se usó $3 \mathrm{mM}$ $\mathrm{MgCl}_{2}, 200 \mathrm{mM}$ dNTPs, 50 mM Tris- $\mathrm{HCl}$ (pH 9,0), 50 $\mathrm{mM} \mathrm{NaCl}, 2,5 \mathrm{U}$ de Taq polimerasa y $1 \mu \mathrm{L}$ de ADN en un volumen total de $25 \mu \mathrm{L}$. Las reacciones consistieron de un paso de denaturación a $94^{\circ} \mathrm{C}$ por $2 \mathrm{~min}, 32$ ciclos de amplificación que corresponden a $30 \mathrm{~s}$ a $94^{\circ} \mathrm{C}$ para denaturación, $30 \mathrm{~s}$ para anillamiento a $62^{\circ} \mathrm{C}$ para los partidores ITS- 2 y $58^{\circ} \mathrm{C}$ para actina canina y $30 \mathrm{~s}$ a $72^{\circ} \mathrm{C}$ para extensión, finalizando con un ciclo de extensión final por 7 min a $72^{\circ} \mathrm{C}^{14}$. Para la identificación molecular de D. repens, se amplificó el gen $12 \mathrm{~S} \mathrm{ADNr}$ usando los par- tidores 12SF/12SR (Tabla 1). Cada reacción de RPC fue amplificada con una mezcla de GoTaq (Promega) y $1 \mu \mathrm{L}$ of $\mathrm{ADN}$ en un volumen total de $15 \mu \mathrm{L}$. El procedimiento de RPC consistió en un paso de denaturación a $94^{\circ} \mathrm{C}$ por 5 min, 35 ciclos de amplificación que corresponden a $30 \mathrm{~s}$ a $94^{\circ} \mathrm{C}$ para denaturación, $30 \mathrm{~s}$ a $48^{\circ} \mathrm{C}$ para anillamiento y $30 \mathrm{~s}$ a $72^{\circ} \mathrm{C}$ para extensión, finalizando con una extensión final de $7 \mathrm{~min}$ a $72^{\circ} \mathrm{C}^{15}$.

Los productos de RPC ( $5 \mu \mathrm{L})$ se visualizaron en un gel de agarosa al 1,5\%. Los fragmentos del tamaño predicho se purificaron usando Mini Kit de Purificación Favorgen (Favorgen Biotech Corp) y secuenciados por Macrogen (Bethesda, Maryland, USA). Los resultados de las secuencias se compararon con las de especies de filarias disponibles en GenBank (National Center for Biomedical Information, E.U.A.)

Análisis filogenético. Se construyó un árbol filogenético por el método de vecino más próximo ("Neighbourjoining") ${ }^{16}$, las matrices de distancia para las secuencias alineadas se calcularon por el método Kimura (two-parameter $)^{17}$ usando el software MEGA 4.0 $0^{18}$. La estabilidad del árbol se estimó por análisis de confianza (bootstrap) de 1.000 replicaciones.

\section{Resultados}

Se observaron microfilarias sanguíneas en 22 de las 100 muestras analizadas (22\%); 16 correspondían a perros sintomáticos $(32 \%)$ y seis a perros asintomáticos $(12 \%)$. Las diferencias entre ambos grupos es significativa $(\mathrm{p}=0,02)$ (Figura 1).

Se estudiaron las características morfológicas de 102 especímenes de microfilarias encontrados en estos 22 perros. En cuatro perros se observaron nematodos que midieron entre 300 y $360 \mu \mathrm{m}$ de largo y 7-8 $\mu \mathrm{m}$ de diámetro, los que podrían corresponder según este parámetro a $D$. repens. Así mismo, se detectaron microfilarias de dimensiones correspondientes a D. dracunculoides (190$247 \mu \mathrm{m}$ de largo y $4-5 \mu \mathrm{m}$ de diámetro) en dos canes, y a $D$. reconditum (260-283 $\mu \mathrm{m}$ de largo y $5 \mu \mathrm{m}$ de diámetro) en

\section{Tabla 1. Partidores utilizados para las amplificaciones de ADN de microfilarias por} RPC en sangre de caninos microfilarémicos

\begin{tabular}{|c|c|c|c|}
\hline Partidor & Región & Secuencia & Referencia \\
\hline DIDR-F1 & ITS-2 & $5^{\prime}$ - AGTGCGAATTGCAGACGCATTGAG -3' & (Rishniw y cols., 2006) \\
\hline DIDR-R1 & ITS-2 & 5' - AGCGGGTAATCACGACTGAGTTGA -3' & \\
\hline Actina F2 & Actina canina & 5' - ACCACTGGTATTGTCATGGACTCTG -3' & \\
\hline Actina R2 & Actina canina & 5'- GCTCTTCTCCAGGGAGGACGA -3' & \\
\hline $12 \mathrm{SF}$ & $12 \mathrm{~S} A \mathrm{ANr}$ & $5^{\prime}$ - ATGTTTTGATTTTTTTTGTAT - $3^{\prime}$ & (Casiraghi y cols., 2006) \\
\hline $12 S R$ & $12 \mathrm{ADNr}$ & $5^{\prime}-$ CCAAATATATATCTGTTTTAAATG -3' & Para este estudio \\
\hline
\end{tabular}




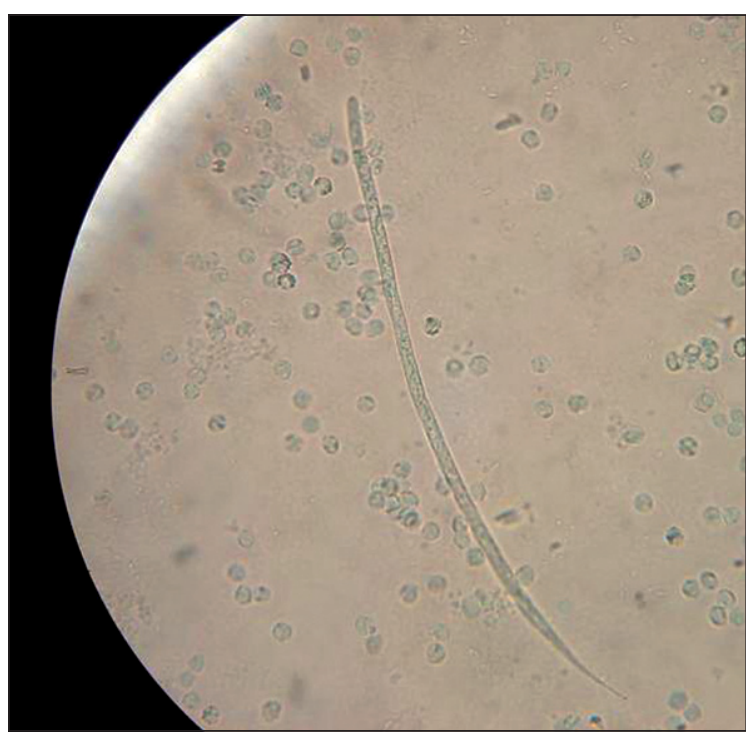

Figura 1. Microfilaria observada con técnica de Knott modificada, en extendido sanguíneo de un perro de la comuna de Lampa (aumento 100X). otros dos. En otros 14 perros y en los cuatro animales que tendrían posibles microfilarias de $D$. repens se observaron conjuntamente parásitos de un tamaño mayor al descrito para las especies que afectan a los cánidos (360-410 $\mu \mathrm{m}$ de largo y 7-8 $\mu \mathrm{m}$ de diámetro), las que fueron denominadas "Dirofilaria sp". Otras características morfológicas de estas microfilarias de mayor longitud fueron similares a las descritas para $D$. repens. No se encontraron especímenes de tamaño correspondiente a D. immitis (290-330 $\mu \mathrm{m}$ de largo y 5-7 $\mu \mathrm{m}$ de diámetro) (Tabla 2).

Catorce de las 22 muestras positivas para microfilarias fueron analizadas para identificación molecular de filaria genérica, entre éstas se encontraban las cuatro que fueron identificadas como posible $D$. repens por parámetros morfológicos. En 12 de estas muestras, la amplificación de la región ITS-2 fue exitosa, resultando un fragmento de 484 pb en 10 muestras y un fragmento de 578 pb en dos. El secuenciamiento del fragmento de $484 \mathrm{pb}$ mostró la mayor cercanía a la única secuencia de $D$. repens disponible en GenBank (AY693808.1) pero con sólo 83,5\% de similitud nucleotídica. Los fragmentos de 578 pb mostraron 99

Tabla 2. Identificación de microfilarias por métodos morfológicos y moleculares en 22 perros de la comuna de Lampa, Chile

\begin{tabular}{|c|c|c|c|c|}
\hline $\begin{array}{l}\text { Perro } \\
n\end{array}$ & $\begin{array}{l}\text { Sintomático } \\
(\mathrm{S} / \mathrm{N})\end{array}$ & $\begin{array}{c}\text { Identificación morfológica } \\
\text { de microfilaremia }\end{array}$ & $\begin{array}{l}\text { Amplificación del gen filarial } \\
\text { ITS-2* y secuenciación }\end{array}$ & $\begin{array}{l}\text { Amplificación del gen de } D \text {. repens } \\
12 S \mathrm{ADNr}^{* *} \text { y secuenciación }\end{array}$ \\
\hline 1 & S & D. reconditum & D. reconditum & NR \\
\hline 2 & N & D. repens y "Dirofilaria sp" & D. repens o especie cercana? & NR \\
\hline 3 & s & D. repens y "Dirofilaria sp" & D. repens o especie cercana? & NR \\
\hline 4 & s & D. repens y "Dirofilaria sp" & D. repens o especie cercana? & NR \\
\hline 5 & s & "Dirofilaria sp" & D. repens o especie cercana? & Cercana a D. repens \\
\hline 6 & s & "Dirofilaria sp" & D. repens o especie cercana? & Cercana a D. repens \\
\hline 7 & N & "Dirofilaria sp" & D. repens o especie cercana? & $N R$ \\
\hline 8 & s & "Dirofilaria sp" & D. repens o especie cercana? & NR \\
\hline 9 & s & D. dracunculoides & Negativa & NR \\
\hline 10 & s & "Dirofilaria sp" & D. repens o especie cercana? & NR \\
\hline 11 & N & D. dracunculoides & Negativa & NR \\
\hline 12 & N & D. repens y "Dirofilaria sp" & D. repens o especie cercana? & NR \\
\hline 13 & S & D. reconditum & D. reconditum & NR \\
\hline 14 & N & "Dirofilaria sp" & NR & NR \\
\hline 15 & s & "Dirofilaria sp" & D. repens o especie cercana? & Cercana a D. repens \\
\hline 16 & S & "Dirofilaria sp" & NR & NR \\
\hline 17 & S & "Dirofilaria sp" & NR & $N R$ \\
\hline 18 & N & "Dirofilaria sp" & NR & NR \\
\hline 19 & S & "Dirofilaria sp" & $N R$ & NR \\
\hline 20 & S & "Dirofilaria sp" & NR & NR \\
\hline 21 & S & "Dirofilaria sp" & $N R$ & NR \\
\hline 22 & s & "Dirofilaria sp" & $N R$ & $N R$ \\
\hline
\end{tabular}




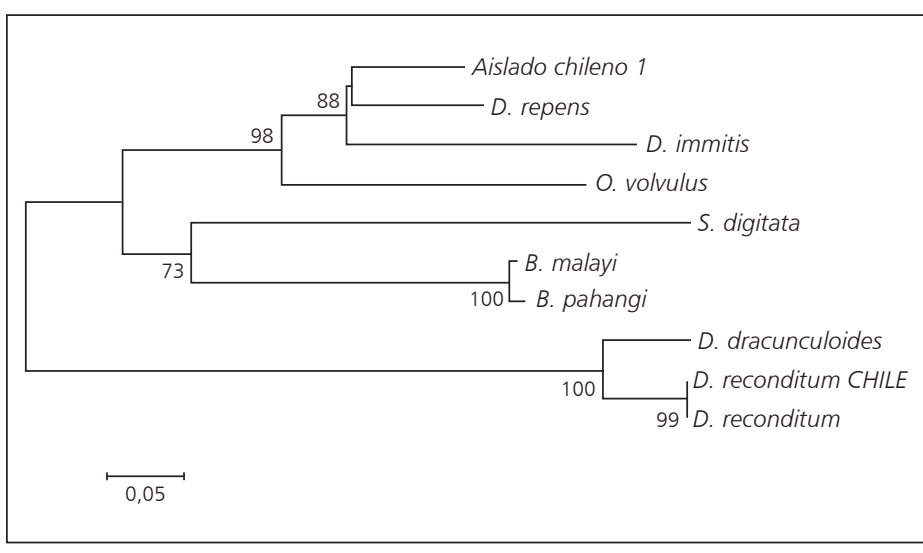

Figura 2. Filogenia de filarias y nemátodos relacionados basada en la región ITS-2. Los números y los nodos corresponden a valores de confianza (bootstrap) después de 1.000 replicaciones; no se muestran valores de confianza menores a 50\%. El árbol filogenético se construyó utilizando el método de vecino más próximo (Neighborjoining) mediante el programa MEGA 4. La barra indica la distancia en sustituciones por nucleótido. Los números de accesos de las secuencias de la región ITS-2 depositadas en GenBank son los siguientes: Dipetalonema reconditum AF217801, Onchocerca volvulus AF228575, Dipetalonema dracunculoides DQ018785, Brugia malayi AY988599, Brugia pahangi AY988600, Setaria digitata EF196091, Dirofilaria repens AY693808 and Dirofilaria immitis AF217800.

y $100 \%$ de similitud con la secuencia de $D$. reconditum AF217801.2 disponible en GenBank. Las dos muestras con microfilaremia por $D$. dracunculoides no amplificaron con estos partidores (Tabla 2). Las dos secuencias de D. reconditum fueron $100 \%$ idénticas entre ellas, lo mismo ocurrió con las cuatro muestras con microfilarias de dimensiones correspondientes a $D$. repens. Las relaciones filogenéticas de estas cepas chilenas con otras dirofilarias se muestran en la Figura 2.

En tres de las cuatro muestras sospechosas de tener microfilarias similares a $D$. repens y con suficiente material extraído remanente, se realizó la amplificación del gen $12 \mathrm{~S} \mathrm{ADNr}$, obteniendo un fragmento esperado de $250 \mathrm{pb}$. Los productos secuenciados de las tres muestras fueron idénticos entre sí y mostraron 95\% de similitud nucleotídica con las secuencias de D. repens depositadas en GenBank (AM779775). Este aislado chileno fue considerado provisoriamente como cercano a D. repens (Tabla 2) y fue incorporado en GenBank con el número de acceso FJ167528. Las relaciones filogenéticas de esta especie chilena con otras dirofilarias se muestran en la Figura 3.

No se observaron diferencias significativas al comparar la frecuencia de síntomas dermatológicos entre los perros con especies de Dipetalomena con aquellos que presentaron la especie cercana a $D$. repens.

\section{Discusión}

La dirofilariasis constituye una infección parasitaria emergente de creciente preocupación en el mundo ${ }^{4}$. Hasta ahora, no se habían publicado estudios que combinen un diagnóstico morfológico y molecular de microfilarias caninas en Chile. Este estudio provee evidencia morfológica que confirmaría la presencia de las dos especies de Dipetalomena previamente descritas en Chile, $D$. dracunculoides y $D$.

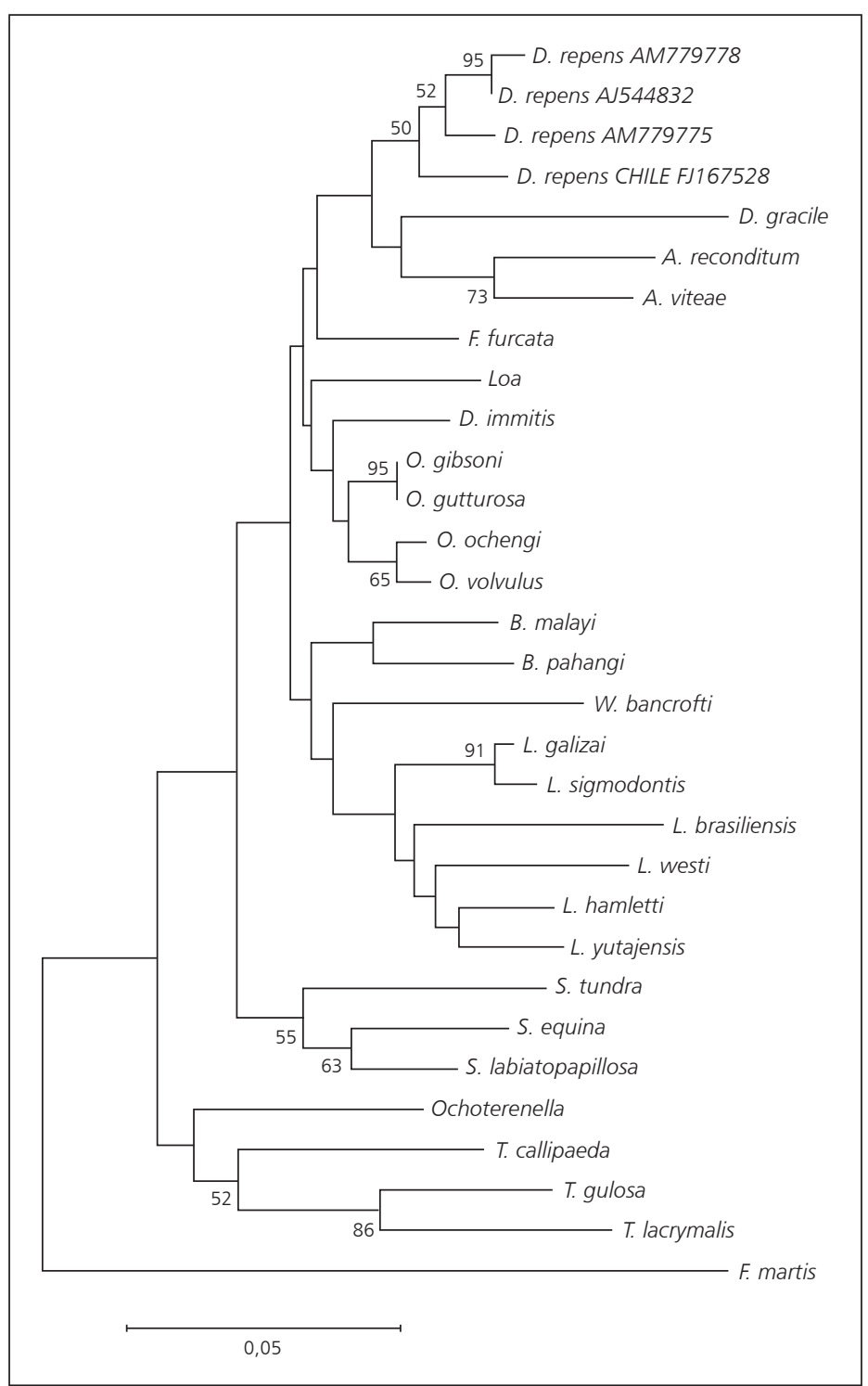

Figura 3. Filogenia de filarias y nemátodos relacionados basada en la secuencia del gen 125 ADNr. Los números y los nodos corresponden a valores de confianza (bootstrap) después de 1.000 replicaciones; no se muestran valores de confianza menores a 40\%. El árbol filogenético se construyó utilizando el método de vecino más próximo (Neighbor-joining) mediante el programa MEGA 4. La barra indica la distancia en sustituciones por nucleótido. Para la construcción del árbol filogenético se utilizaron las secuencias del gen 12S ADNr depositadas en GenBank con los siguientes números de acceso: Acanthocheilonema reconditum AJ544853; Acanthocheilonema viteae AJ544852; Brugia malayi AJ544843; Brugia pahangi AJ544842; Dipetalonema gracile AJ544854; Dirofilaria immitis AJ544831; Dirofilaria repens AJ544832-AM779775-AM779778; Filaria martis AJ544855; Foleyella furcata AJ544841; Litomosa westi AJ544851; Litomosoides brasiliensis AJ544850; Litomosoides galizai AJ544849; Litomosoides hamletti AJ544847; Litomosoides sigmodontis AJ544848; Litomosoides yutajensis AJ544846; Loa loa AJ544845; Ochoterenella sp. 56cv AJ544836; Onchocerca gibsoni AJ544837; Onchocerca gutturosa AJ544838; Onchocerca ochengi AJ544839; Onchocerca volvulus AJ544840; Setaria equina AJ544835; Setaria labiatopapillosa AJ544834; Setaria tundra AJ544833; Wuchereria bancrofti AJ544844; Thelazia callipaeda AJ544858; Thelazia gulosa AJ544857n y Thelazia lacrymalis AJ544856. 
reconditum ${ }^{8,9}$, sumado a la confirmación molecular de esta última. Además, se identificó morfológicamente la presencia microfilarias de longitud similar a $D$. repens, especie no descrita en el país. Este hallazgo no pudo ser confirmado molecularmente debido a que la homología nucleotídica del fragmento secuenciado no fue mayor a 95\% con las escasas secuencias de D. repens disponibles para comparación. Este hecho, sumado a la observación de que hubo una importante proporción de microfilarias morfológicamente similares a $D$. repens, pero de un tamaño mayor al descrito para esta especie, nos conduce a plantear la existencia de otra especie de dirofilaria en Chile o de una variante relacionada a $D$. repens. Esta dirofilaria fue más frecuente que las especies de Dipetalomena no patógenas, afectando a $10 \%$ de los perros estudiados.

Coincidentemente con este hallazgo, en un estudio previo chileno se encontró algunas microfilarias de un tamaño mayor al descrito en la literatura para las especies caninas y similar a las aquí reportadas ${ }^{8}$. Los autores indican similitud de estas microfilarias con Dirofilaria striata; sin embargo, esto parece poco probable por tratarse de una especie descrita en félidos silvestres, pudiendo tratarse de la misma especie que estamos reportando.

Los requisitos climáticos y ambientales para el exitoso desarrollo de dirofilarias en un área determinada incluyen temperaturas ambientales sobre los $18-20^{\circ} \mathrm{C}$ y la abundancia de mosquitos transmisores competentes ${ }^{19}$. El mencionado límite de temperaturas se cumple por varios meses en muchas áreas de Chile, incluyendo el área bajo estudio en el presente trabajo; lo mismo ocurre con la presencia y abundancia de mosquitos Culex en la mayoría del país y en particular en la zona geográfica del estudio $^{10}$. La densidad de mosquitos y la abundancia de perros microfilarémicos son los factores de riesgo más importantes para la infección humana ${ }^{2,20}$. Se ha estimado que con $7 \%$ o más de perros microfilarémicos en un área, el riesgo para infección humana es significativo ${ }^{20}$. Esto indica que con el porcentaje de perros microfilarémicos encontrado en la zona estudiada, es bastante factible la ocurrencia de casos humanos. El caso humano recientemente reportado en nuestro país, ocurrido en una niña habitante de una comuna muy cercana a la del estudio ${ }^{11}$, apoya esta conclusión.

Fue notorio el alto porcentaje de perros parasitados con microfilarias en este estudio (22\%), de los cuales la gran mayoría $(18 / 22)$ presentaba $D$. repens y/o una especie cercanamente relacionada. Las publicaciones nacionales previas mostraban cerca de $30 \%$ de infestación, pero a diferencia del presente estudio, la casi totalidad de los especímenes correspondían a Dipetalomena ${ }^{8,9}$. Destaca asimismo en nuestro estudio la significativa mayor frecuencia de dirofilariasis en perros con síntomas dermatológicos. En dos grandes estudios de infección por $D$. repens en canes, el principal síntoma asociado con infección fue la dermatitis prurítica; los nódulos subcutáneos, que constituyen el principal síntoma de la infección en humanos parecen ser infrecuente en perros ${ }^{5,6}$. En un estudio reciente en 100 perros con $D$. repens se describen variadas manifestaciones dermatológicas, siendo las más frecuentes: prurito, eritema, pápulas, alopecia focal o multifocal ${ }^{6}$. Un autor reporta que 4/9 perros microfilarémicos y asintomáticos al momento del diagnóstico desarrollaron dermatitis prurítica en el lapso de 5 meses $^{5}$. Similares síntomas se han descrito ocasionalmente en asociación a otras especies de Dirofilaria, como D. immitis e incluso, D. reconditum ${ }^{6}$. Si bien nuestros resultados no permiten atribuir con certeza los síntomas dermatológicos que presentaban los animales a la dirofilariasis, la significativa mayor prevalencia de microfilarias en perros con sintomáticos sugiere algún rol etiológico de esta parasitosis en estas manifestaciones dermatológicas. En el presente estudio se observaron microfilarias en 6 de 50 perros asintomáticos, proporción similar a la reportada en un área conocidamente endémica a $D$. repens (norte de Italia $)^{5}$. Este hecho destaca la relevancia epidemiológica de nuestros hallazgos, ya que se pudo constatar que la filariasis canina parece estar ya bien establecida en esta zona de Chile.

Se requieren estudios moleculares complementarios para precisar si los especímenes morfológicamente similares a $D$. repens pero de mayor longitud constituyen una variante o una nueva especie cercana a ella. Asimismo, queda por estudiar la extensión geográfica de esta parasitosis canina en el resto del país.

Nuestros resultados deberían alertar a los médicos clínicos a tener presente esta parasitosis, principalmente en pacientes con nódulos subcutáneos; en especial considerando que las áreas con reportes de dirofilariasis humana en el mundo se han expandido en forma importante, sumándose a las zonas tradicionalmente consideradas endémicas al menos 35 nuevos países o áreas geográficas en la última década ${ }^{4}$.

Agradecimientos: A Aníbal San Martín del Departamento de Higiene y Zoonosis de la Municipalidad de Lampa, Chile, por su ayuda en el reclutamiento y toma de muestra de perros para el estudio.

El estudio fue apoyado en parte por el Laboratorio de Infectología y Virología Molecular, Pontificia Universidad Católica de Chile.

\section{Resumen}

Introducción: A la fecha no hay datos concluyentes en Chile respecto a la presencia de dirofilariasis zoonótica en perros. Objetivos: Identificar la presencia de dirofilarias en sangre de perros de una comuna semi-rural cercana a 
Santiago y comparar su frecuencia en animales con y sin manifestaciones dermatológicas. Material y Métodos. Se examinó un frotis sanguíneo de 100 perros en busca de microfilarias mediante observación microscópica (técnica de Knott modificada). Cincuenta perros presentaban síntomas o signos dermatológicos que se han asociado a esta parasitosis y 50 eran asintomáticos. Se amplificaron los genes ITS-2 y 12s ADNr de filarias en las muestras con microfilarias al frotis, secuenciando los fragmentos amplificados. Resultados: Se observaron microfilarias en 22 perros $(22 \%), 16 / 50(32 \%)$ sintomáticos y $6 / 50$ $(12 \%)$ asintomáticos $(\mathrm{p}=0,02)$. Morfológicamente, la mayoría de las microfilarias observadas fueron similares a D. repens; sin embargo, una gran proporción mostró un tamaño mayor al descrito para esta especie. Las secuencias nucleotídicas de los genes amplificados mostraron una homología no mayor al $95 \%$ con las secuencias de $D$. repens disponibles para comparación. Se identificaron además dos especies poco patógenas, D. reconditum por morfología y secuenciación genética y $D$. dracunculoides por morfología. Conclusiones: Los resultados indican la existencia de una nueva especie de Dirofilaria cercanamente relacionada a $D$. repens o de una variante de esta especie.

\section{Referencias bibliográficas}

1.- Georgi, J. Nemátodos. En: Parasitología en Clínica Canina. D.F, México, McGraw-Hill 1994, pp: 156-216.

2.- Muro A, Genchi C, Cordero M, Simón F. Human dirofilariasis in the European Union. Parasitol Today 1999; 15: 386-9.

3.- Pampiglione S, Rivasi F. Human dirofilariasis due to Dirofilaria (Nochtiella) repens: An update of world literature from 1995 to 2000. Parassitologia 2000; 42: 231-54.

4.- Simón F, Morchón R, González-Miguel J, Marcos-Atxutegi C, Siles-Lucas M. What is new about animal and human dirofilariasis? Trends Parasitol 2009; 25 (9): 404-9.

5.- Tarello W. Cutaneous lesions in dogs with Dirofilaria (Nochtiella) repens infestation and concurrent tick-borne transmitted diseases. Vet Dermatol 2002; 13: 267-74.

6. Tarello W. Clinical aspects of dermatitis associated with Dirofilaria repens in pets: A review of 100 canine and 31 feline cases (19902010) and a report of a new clinic case imported from Italy to Dubai. J Parasitol Res 2011, 578385, Epub 2011 Dec 13.

7.- Labarthe N, Guerrero J. Epidemiology of heartworm: what is happening in South
America and Mexico? Vet Parasitol 2005; 133: 149-56.

8.- Alcaíno H, Gorman T R, Puelma M. Filariasis canina en Chile. Arch Med Vet 1984; 16 : 67-73.

9.- Alcaíno H, Monasterio X, Gorman TR. Canine filaridosis in the V Region of Chile. Parasitol Día 1995; 19: 143-5.

10.- Almiron W R, Harbach R E. Taxonomy and biology of Culex (Culex) maxi Dyar (Diptera: Culicidae) in South America. Mem Inst Oswaldo Cruz 1996; 91: 579-88.

11.- Pérez L C, Arce J D. Nódulos parasitarios cutáneos: Estudio ultrasonográfico de tres casos poco frecuentes en la edad pediátrica. Rev Chil Radiol 2007; 13: 163-8.

12.- Genchi C, Venco L, Genchi M. Guideline for the laboratory diagnosis of canine and feline Dirofilaria infections. In: C. Genchi, L. Rinaldi and G. Cringoli, Editors, Dirofilaria immitis and D. repens in dog and cat and human infections, Rolando Editore, Naples 2007, pp. 138-44.

13.- Nuchprayoon S, Junpee A, Nithiuthai S, Chungpivat S, Suvannadabba S, Poovorawan Y. Detection of filarial parasites in domestic cats by PCR-RFLP of ITS1. Vet Parasitol 2006; 140: 366-72.

14.- Rishniw M, Barr S C, Simpson K W,
Frongillo M F, Franz M, Domínguez Alpizar J L. Discrimination between six species of canine microfilariae by a single polymerase chain reaction. Vet Parasitol 2006; 135: 303-14.

15.- Casiraghi M, Bazzocchi C, Mortarino M, Ottina E, Genchi C. A simple molecular method for discriminating common filarial nematodes of dogs (Canis familiaris). Vet Parasitol 2006; 141: 368-72.

16.- Saitou N, Nei M. The neighbor-joining method: a new method for reconstructing phylogenetic trees. Mol Biol Evol 1987; 4: 406-25.

17.- Kimura M. A simple method for estimating evolutionary rates of base substitutions through comparative studies of nucleotide sequences. J Mol Evol 1980; 16: 111-20.

18.- Tamura K, Dudley J, Nei M, Kumar S. MEGA4: Molecular evolutionary genetics analysis (MEGA) software version 4.0. Mol Biol Evol 2007; 24: 1596-9.

19.- Trotz-Williams L A, Trees A J. Systematic review of the distribution of the major vectorborne parasitic infections in dogs and cats in Europe. Vet Rec 2003; 152: 97-105.

20.- Sabu L, Devada K, Subramanian H. Dirofilariosis in dogs and humans in Kerala. Indian J Med Res 2005; 121: 691-3. 